\title{
Reviewer Summary
}

Journal of Pharmacy Technology

2017, Vol. 33(I) 40-4

(C) The Author(s) 2016

Reprints and permissions:

sagepub.com/journalsPermissions.nav

DOI: $10.1|77 / 8755| 22516687493$

journals.sagepub.com/home/pmt

(S)AGE

The individuals listed below reviewed manuscripts for the Journal of Pharmacy Technology between November 1, 2015 and November 1, 2016. We are glad to take this opportunity to acknowledge our referees and to thank them for their time and effort.

Abdelfattah, Samir

Abdelrahim, Mohamed

Adams, Alex

Al-Badriyeh, Daoud

Alvarez-Risco, Aldo

Alvarez-Risco, Aldo

Anksorus, Heidi N

Aslani, Parisa

Athans, Vasilios

Bansal, Vinod K

Battaglia, Jessica N

Beigi, Pooya

Bernknopf, Allison

Bertino, Jr., Joseph S.

Bhatt, Snehal H

Black, Robin

Bohm, Nicole M

Borchert, Jill S

Bright, David

Brinkman, Mary

Britton, Emily

Brown, Sherrill J

Brown, Verender

Brunetti, Luigi

Burgess, Sarah

Campbell, Kristen Bova

Chim, Christine

Chudow, Melissa

Chui, Sai Ho

Cierniak, Kayla

Cimino, Christo

Clark, Nathan

Davydov, Liya

Dering-Anderson, Allison

Dominguez-Salazar, Krista

Dunham, Marissa Waldman

Durham, Spencer

Eckhardt, Douglas

Eudaley, Sarah

Evans, Elizabeth Sebranek

Everson, Nathan

Eyler, Rachel F

Faris, Janie

Farthing, Kate

Fox, Lanae
Freeman, Katherine

Gates, Brian J

Geddis, Janell

Griffin, Brooke

Halikman, Jennifer

Hardin, Heather

Hohmeier, Kenneth

Hoke, Kathleen

Huang, Melody H

Hughes, Christine A

Huston, Sally A

James, Renee

Jensen Bender, Wendy

Jensen, Leon K

Justis, Leanne

Kang-Birken, Sunghe Lena

Kee, Vicki R

Keeshin, Susana

Kehr, Heather A

Kelling, Sarah

Keresztes, Jan M

Khan, Shamima

Kline, Jonathan

Knockel, Laura

Kopcza, Kathleen B

Kugler, Anne

Kuhl, Stephanie

Lafitte, Elizabeth

Lamprecht, Donald G

LaVance, Anne

Lee, Bernard Ryan

Leung, Marianna

Levien, Terri L

Lewis, Kimberly Pershun

Li Ying, Huang

Lopez, Julio R

LoTurco, Michelle

Lui, Mimi

Lukasiewicz, Ronald H.

MacCallum, Colleen

Magedman, Grace

Makowski, Charles

Manville, Nathan

Martin, Linda Gore

McCoy, Cheryl 
Mehta, Hemalkumar

Meyer, Allison

Miano, Todd

Nebelsick, Kyle

Niyyati, Seyed

Norris, LeAnn

Olsommer, Melissa

Park, Jiehye

Patanwala, Asad

Patel, Hansita

Pattie, Stacey Baker

Peikari, Hamid Reza

Ponder, Richard

Priano, James

Prudencio, Jarred

Qato, Danya

Raake, Sarah Elizabeth

Reed, Erica

Rice, Kathryn

Rinka, Joe

Rivard, Kaitlyn

Roane, Teresa E

Rogers, Jennifer

Rumore, Martha

Salerno, David

Scalese, Michael

Schafer, Jason

Schmelz, Andrew N

Schulz, Martin

Shin, Maria

Smith, Jennifer G

Smith, Kelly
Smoot, Thomas

Sommer, Christine D

Stafford, Rachel

Stoner, Steven

Stefanadis, Constantine

Streetman, Daniel S

Stulock, Rachel

Stultz, Jeremy

Tran, Steven

Truong, Christine

Turley, James C

Turner, Adrian

Vander Ploeg, Sara

Verheyden, Collin

Vick, Jarrod

Wagner, Jamie

Wang, Sheng

Westerlund, LO Tommy

Williamson, Bobbie

Wilson, Debbie

Wilson, Dustin

Wilson, James Paul

Wilson, Jennifer

Wright, Eric

Yamaki, Jason

Yancey, Abigail

Yang, Esther

Yee, Deanna

Yoon, Jihyun

Yuksel, Nese

Yunker, Nancy

Zaiken, Kathy 